\title{
An Adaptive Image Registration Technique to Remove Atmospheric Turbulence
}

\author{
Akshay Patel ${ }^{1}$, Dippal Israni ${ }^{1, *}$, Nerella Arun Mani Kumar ${ }^{2}$, Chintan Bhatt $^{1}$ \\ ${ }^{1} U$ and $P$ U. Patel Department of Computer Engineering, CSPIT, CHARUSAT \\ 2 Central Research Laboratory, Bharat Electronics, Bangalore, India
}

\begin{abstract}
Turbulence/Heat Scintillations is the change that arises in the refraction index of air with the temperature. The distortion created by the atmospheric turbulence is proportional to the distance between object and camera. In last few years, several approaches have been proposed to estimate and eliminate geometric distortion and blur. In this paper, a novel technique is proposed that improves the visual quality of video sequences affected by atmospheric turbulence. The proposed method is based on adaptive control grid interpolation (CGI). CGI approximates accurate motion vectors to generate a geometrically correct frame using certain reference frames. For high scintillation sequences, CGI doesn't mitigate scintillations completely. The new methodology is proposed with updated trajectory estimation. The proposed method can effectively reduce the influence even for high atmospheric turbulence. Experimental results also prove that proposed approach is time efficient compare to traditional CGI.
\end{abstract}

Keywords Heat Scintillation, Atmospheric Turbulence, Motion Vector Calculation, Image Registration, Blind Iterations, Optical Flow.

AMS 2010 subject classifications 68U10

DOI: $10.19139 /$ soic.v7i2.432

\section{Introduction}

The visual quality of long rang imagery capturing can be influenced by various types of atmospheric distortion [1]. Hot air, fog, and haze are the primary reason behind geometric distortion and space and time-arying blur [1][2]. Captured frames can be mathematically represented as

$$
f_{k}=D_{k}+B_{k}+I_{k}+\epsilon
$$

Where $f_{k}$ represents $k^{t h}$ observed frame, $D_{k}$ and $B_{k}$ denote the deformation matrix and the blurring matrix respectively for the $k^{t h}$ frame, $I_{k}$ denotes original image without any effect of turbulence and $\epsilon$ denotes the noise [3]. Heat scintillations are reliable on certain parameters like temperature, the speed of wind, the distance between object and camera, and time. Two major techniques are mainly used for mitigation. First one is based on frame reconstruction using sequential video frames [4][5]. This method uses non-rigid image registration technique to register each observed frames with using turbulence free reference frame [3][4][5]. Non-registration based Sobolev Gradient and Laplacian approach is proposed by Y. Lou et al et.al to stabilize the video sequence [6]. To mitigate the effect cause by turbulence is necessary for long distance image capturing. This is useful for surveillance, defence and in many commercial applications.Turbulence caused by atmosphere leads to most of the oscillation in the video

\footnotetext{
*Correspondence to: Dippal Israni (Email: dippalisrani.ce@ charusat.ac.in). U and P U. Patel Department of Computer Engineering, CSPIT, CHARUSAT, Changa (388421).
}

ISSN 2310-5070 (online) ISSN 2311-004X (print)

Copyright (C) 2019 International Academic Press 
sequence. There comes a need to solve the problem related to it as it has a vast scope of research issues. Most of the work done in removing turbulence effect from the video sequence corrects the geometric distortion only. There is a need to stabilize the video sequence with a clear view of the scene for visible and infrared bands with minimal computations.

This paper presents the blind technique to estimate required iterations using Mean Square Error to mitigate turbulence and retrieve the clear view of the scene.

\section{Related Work}

Image registration based techniques are highly used for reconstruction of the turbulent affected frame [2]. Image processing is generally used for estimating motion and shift between sub sequent images of the video or time deleted images [7]. There are lists of traditional restoration algorithms[8][9], However they are not useful for mitigation of atmospheric degradation. In image registration, sequential video frames are used for computing the time-varying distortion by exploiting quasi-periodicities in a motion field. Several registration techniques are presented such as GFATR (Generalized First Average than Register) registration [10]. In this approach single reference frame is used to register all observed frames. Also, reference frame is selected having negligible geometric distortion as well as time-varying blur. FRTAAS (First Register than Average and Subtract) is updated method of FATR in which registered frames are used to update reference frame [10]. Elastic image registration is proposed by $\mathrm{J}$ Kybic et al. that incorporates geometric mapping that is locally affine and also globally smooths [11]. Elastic image registration technique can also be used in medical imaging is proposed by S. Periaswamy et al. [12]. The CLEAR algorithm proposed by N Anantrasirichai et al. [1][13], stabilizes the video by aligning (by translation) the centers of corresponding regions of interest (ROI). High-quality frames are selected for further processing on the basis of sharpness, intensity similarity, and ROI size. Another technique to improve the resolution of video frames is by using bilinear interpolation [14][15]. CGI is one of the techniques that use bilinear interpolation. In CGI, represented by Sullivan et.al [16], motion detection performed by spatial image transformation. Segmenting image into the small square region with corner control points. Three bilinear interpolations per pixel, two for horizontal and vertical part of the motion vector, the third one is for the predicted intensity of a pixel in previous decode frame [16]. Sequential Control Grid Interpolation (CGI) that uses the same concept using several adjacent reference frames is proposed by D. H. Frakes et.al [17]. Still, this approach lacks in mitigating sequences that have a high amount of scintillation. Also, the traditional approach has high CPU time.

\section{Proposed Approach}

The main challenge that traditional CGI face is that partial scintillation is still left out and (with) higher CPU computations. To remove both disadvantages this paper proposes a novel CGI based technique that adaptively updates trajectory estimation. A block diagram that includes the basic flow of the proposed system is as described in fig 1 . The distorted video sequence is only input to mitigate the problem. Spatial transformation is applied to the

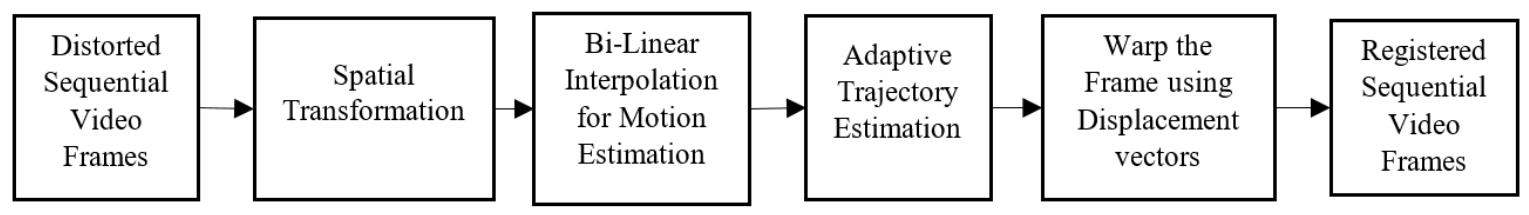

Figure 1. Proposed System

video sequences. The motion vector is being calculated for small continues square region. Trajectories for all the pixels are calculated using the motion vectors. A block diagram that includes the flow of the proposed approach is as described in fig 2. Details of each step in algorithm are described below. 


\subsection{Distorted Sequential Video Frames}

Most commonly, High-resolution visible band video capturing is preferable for applications like surveillance and defense. The removal of geometrical distortion is not potential using one observed video frame, Hence distorted video sequences are used to compensate distortion. In related work certain approaches are described that performs registration by converting sequences into grayscale. Here, a standard dataset of Open Turbulent Image Set (OTIS) is used for testing the proposed approach that contains visible band sequences [18]. In this research the electric fields inside the insulation of the construction were determined from a boundary element method simulation: along a circle among the phases and the tank, along vertical line starting from the bottom of the tank, along line connecting the centers of conductor phases, and around the conductor. The electric field calculation is two dimensional.

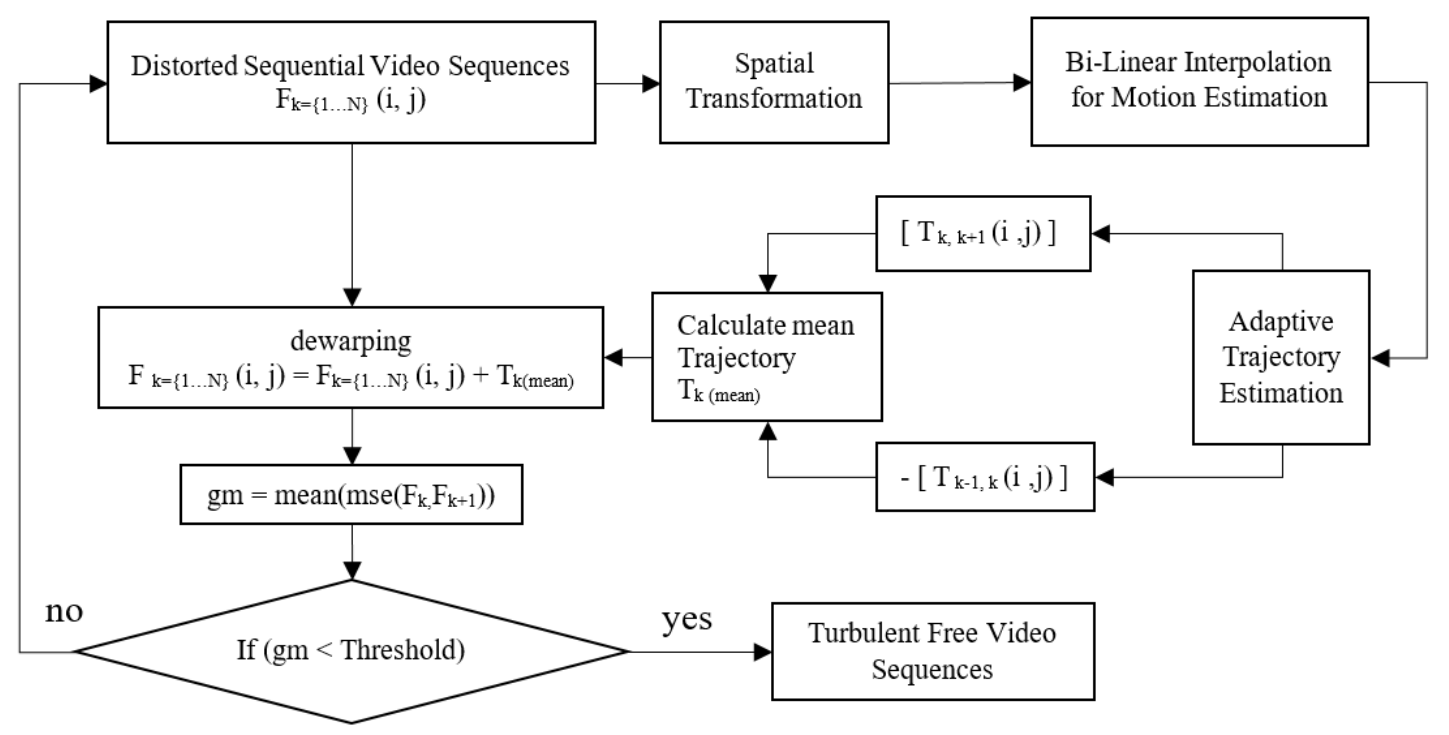

Figure 2. System flow of proposed approach

\subsection{Spatial Transformation}

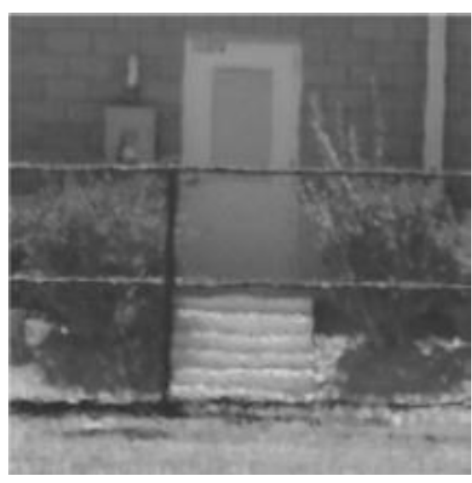

(a)

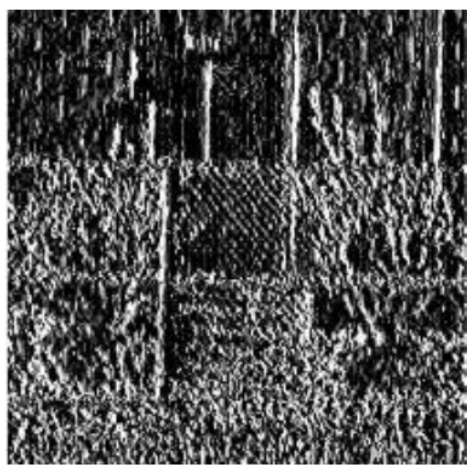

(b)

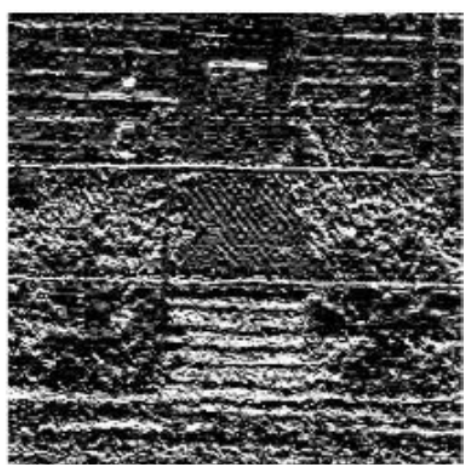

(c)

Figure 3. (a) Gray scale frame, (b) Horizontal convolution of (a) and (c) Vertical convolution of (a) 
Non-label based spatial transformation is used to minimize some index of difference between the target image and reference image [19]. Two-dimensional basic derivative filters are used to convolve image horizontally and vertically. As shown in Fig. 3 The grayscale frame is used to find the spatial transformation.

\subsection{Bi-linear Interpolation for Motion Estimation}

The purpose of motion estimation technique is to allow similar features of the different images to be registered [20]. In CGI, registration is performed among the small and same size blocks of the target and reference frames.

$$
\begin{aligned}
& F_{1}[i, j]=F_{0}\left[i+d_{1}[i, j]+d_{2}[i, j]\right] \\
& d_{1}[i, j]=\alpha_{1}+\alpha_{2} i+\alpha_{3} j+\alpha_{4} i j \\
& d_{2}[i, j]=\beta_{1}+\beta_{2} i+\beta_{3} j+\beta_{4} i j
\end{aligned}
$$

The relationship between pixels among the block in frame $F_{0}[i, j]$ and $F_{1}[i, j]$ is described in (2). Here $i$ and $j$ represent the pixel coordinates, $d_{1}[, j]$ and $d_{2}[i, j]$ represent horizontal and vertical displacement of the pixel between two frames $F_{0}$ and $F_{1}, \alpha$ and $\beta$ are parameters which are determined for each block B. $\alpha$ and $\beta$ can be calculated by minimizing.

$$
\sum_{[i, j] R}\left[F_{1}[i, j]=F_{0}\left[i+d_{1}[i, j]+d_{2}[i, j]\right]\right]^{2}
$$

Here we can use first-order Taylor series approximation

$$
\sum_{[i, j]}\left[F_{0}[i, j]-F_{1}[i, j]-\frac{\delta F_{1}[i, j]}{\delta i} d_{1}[i, j]-F_{1}[i, j]-\frac{\delta F_{1}[i, j]}{\delta j} d_{2}[i, j]\right]^{2}
$$

Which discard the high order terms from (3). Bilinear parameters of the calculated motion vector are used to recover the displacement of the pixels within the region.

\subsection{Adaptive Trajectory Estimation}

Several approaches are proposed in [2][17]. One of them is to estimate the final trajectory of each new frame using the trajectory of the previous frame. This is Mathematical represented as

$$
\begin{aligned}
& T\left(i, j, t_{0}\right)=F_{0}(i, j) \\
& T\left(i, j, t_{0}-1\right)=T\left(i, j, t_{0}\right)+v_{t_{0}, t_{0}-1}(i, j) \\
& \vdots \quad \vdots \\
& T\left(i, j, t_{0}-n\right)=T\left(i, j, t_{0}-n+1\right)+v_{t_{0}, t_{0}-n}(i, j)
\end{aligned}
$$

Where $F_{0}$ denotes target frame, $T$ represents trajectory, $v$ represents motion vector between the target frame and reference frames. Total $n$ trajectories for single target frame where $n$ denotes the number of frames used as reference frame [2][17]. This method repeats the error of trajectory calculation for all compounded frames [2]. Another technique is to find the target frame trajectory to fix the target frame. This will increase the computational load.

$$
\begin{gathered}
T\left(i, j, t_{0}+n\right)=F_{0}(i, j)+v_{t_{0}, t_{0}+n}(i, j) \\
\vdots \\
\begin{array}{c}
T \\
T\left(i, j, t_{0}+1\right)=F_{0}(i, j)+v_{t_{0}, t_{0}+1}(i, j)
\end{array} \\
T\left(i, j, t_{0}\right)=F_{0}(i, j) \\
T\left(i, j, t_{0}-1\right)=F_{0}(i, j)+v_{t_{0}, t_{0}-1}(i, j) \\
\vdots \\
\begin{array}{c}
T \\
\left(i, j, t_{0}-n\right)=F_{0}(i, j)+v_{t_{0}, t_{0}-n}(i, j)
\end{array}
\end{gathered}
$$


The motion vector is required to be calculated each time. Therefore, the error-propagation problem is solved, but the computation is greatly increased. This happens as target frame shifts and each motion field with the previous frame is required to be recomputed [18]. The total number of trajectories is $2 \times n$, due to change in selecting reference frame. Consecutive $(2 \times n)+1$ number of frames used as reference except $(n+1)^{t h}$ frame which is target frame [2]. From input video sequences, total $2 \times n$ number of video frames will never be turbulent free. The first and last $\mathrm{n}$ frames in the video sequence will be used as a reference frame but not as a target frame. Here, a novel trajectory estimation technique is proposed, which mitigates both arrived problems in previous techniques. For calculating an efficient trajectory for target frame, calculate all trajectory of the sequential turbulent frame using its next frames as a reference frame. Mathematical representation of proposed technique is as follow

$$
\begin{gathered}
T_{0}\left(i, j, t_{0}\right)=F_{0}(i, j)+v_{t_{0}, t_{1}}(i, j) \\
T_{1}\left(i, j, t_{1}\right)=F_{1}(i, j)+v_{t_{1}, t_{2}}(i, j) \\
\vdots \\
\begin{array}{c}
T_{k}\left(i, j, t_{k}\right)=F_{k}(i, j)+v_{t_{k}, t_{k+1}}(i, j) \\
\vdots \\
T_{n-1}\left(i, j, t_{n-1}\right)=F_{n-1}(i, j)+v_{t_{n-1}, t_{n}(i, j)}
\end{array}
\end{gathered}
$$

Here, Total $n$ frames of input video frames. Total $n-1$ number of trajectories is calculated for $n$ turbulent frames. Each trajectory is being calculated using its next frame as a reference frame. In process of calculating $T_{k}$, the $k^{t h}$ frame is target frame and $(k+1)^{t h}$ is the reference frame. As shown in (7), $T_{0}$ to $T_{n-1}$ are trajectories for $n$ number of turbulent frames. To calculate the final trajectory matrix of each frame except first and last frame, mean is calculated using two continues trajectory metrics. The final trajectory of the $k^{\text {th }}$ frame using mean trajectory of $\left[T_{k, k+1}(i, j)\right]$ and $\left[-\left[T_{k, k-1}(i, j)\right]\right]$. Here, the sum matrix is divided by 3 to find mean, due to three frames are involved to calculate trajectory.

\subsection{Image Warping}

Warping is the process to digitally manipulate the image. In brief, the points are projected to points without varying its colors. In this approach, warping is used to correct distortion. Pixels of input video frames are horizontally and vertically mapped using trajectories of each pixel, which generates the geometrically corrected frames - Registered sequences. In case of color sequences, after estimating trajectory, warping is applied on all three planes (r-g-b) of the target frame. This simple technique is useful because the distortion of colors is less influence on human visual perception.

\subsection{Threshold C Blind Iteration}

The global MSE (Mean Square Error)[21] is calculated of registered sequences. MSE is used to measure the average of squares of the errors. Global MSE of $n$ registered frames is calculated by taking the mean of an array of MSE values. The MSE of an array is of $n-1$ size which contains MSE between consequences frames. The global MSE will be compared with a threshold value. If it is smaller than the threshold, the registration process will continue by using registered frames as input video sequences. If global MSE is larger than the threshold value, iteration will be stopped.

\subsection{Turbulence Free Video Sequences}

After getting global MSE which is lesser than the threshold, the registered frames will be final output. The output video sequence is achieved resultant turbulence free video sequence on the basis of the threshold value. 


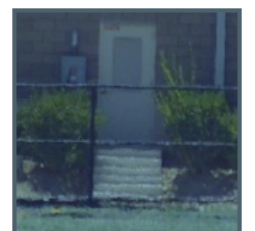

(a) $9^{\text {th }}$ Observed Frame

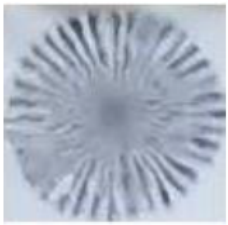

(d) $9^{\text {th }}$ Observed Frame

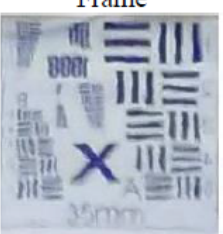

(g) $9^{\text {th }}$ Observed Frame

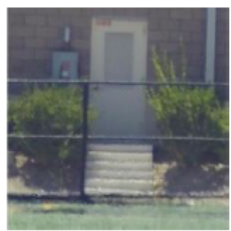

(b) Original CGI

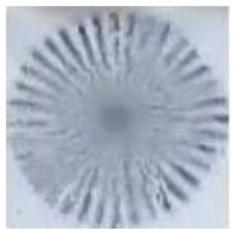

(e) Original CGI

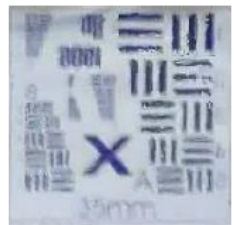

(h) Original CGI

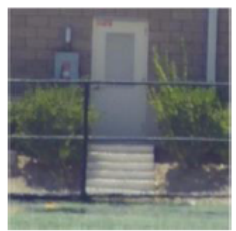

(c) Adaptive CGI

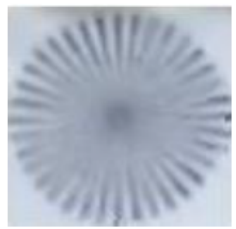

(f) Adaptive CGI

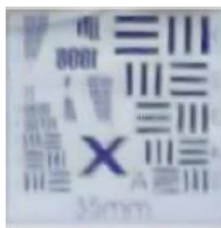

(i) Adaptive CGI

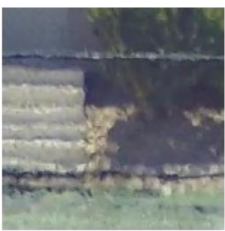

(a1) zoom of a

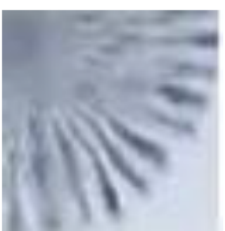

(di) Zoom of d

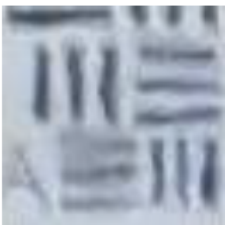

(g1) Zoom of d

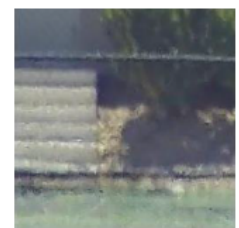

(b1) zoom of b

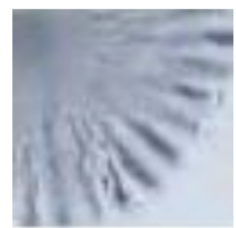

(e) Zoom of e

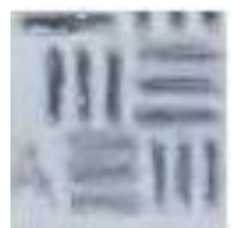

(hi) Zoom of e

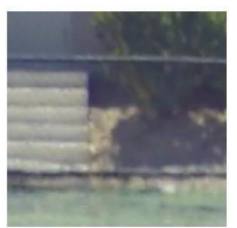

(c1) zoom of c

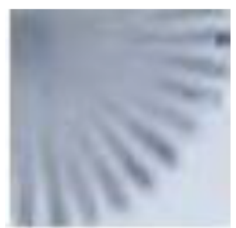

(fi) Zoom of $f$

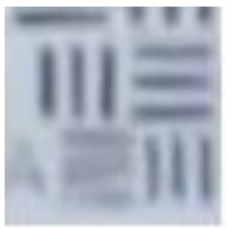

(ii) Zoom of i

Figure 4. (a)(d)and (c) $9^{\text {th }}$ observed frames from dataset sequences, (b),(e) and (h) output results of (a),(d) and (g) respectively using CGI, (c),(f) and (i) output results of (a),(d) and (g) respectively using ACGI. (a1) to (i1) is zoom of (a) to (i) respectively

\section{Experiment and Simulation}

Adaptive CGI approach has been tested using three sequences of a state of the art dataset C OTIS [18]. These datasets are simulated datasets containing sequences of optical observations made through atmospheric turbulence.

\begin{tabular}{|c|c|c|c|c|}
\hline Video & Dataset & band & Number of frames & Resolution \\
\hline Door & OTIS & Visible Band & 25 & 520520 \\
Pattern 6 & OTIS & Visible Band & 25 & 113109 \\
Pattern 11 & OTIS & Visible Band & 25 & 183172 \\
\hline
\end{tabular}

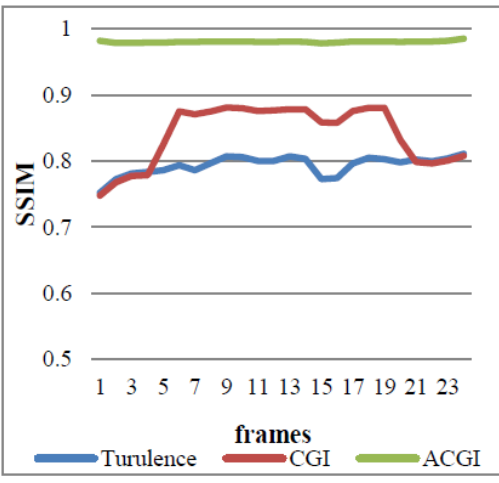

(a) Door squences

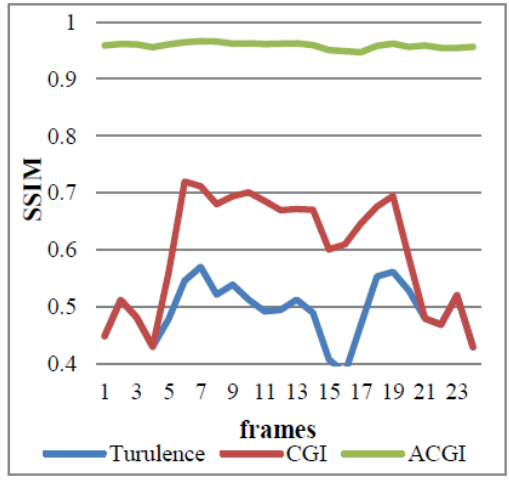

(b) Pattern 6 sequncces

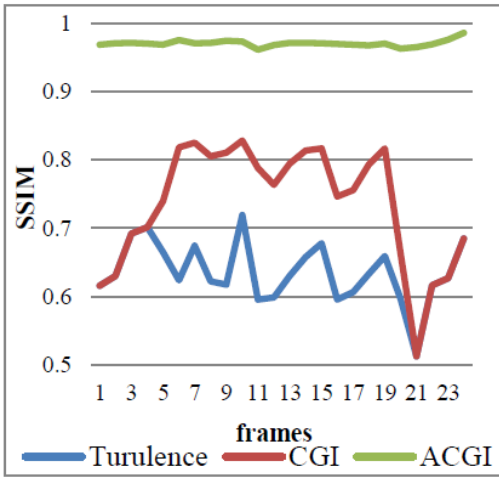

(c) Pattern 11 sequence

Figure 5. (a), (b) and (c) SSIM between consecutive frames in Turbulence, CGI and ACGI of door, pattern 6 and pattern 11 
The results represented in fig. 4 shows that CGI mitigate the turbulence but the intensity is more accurate in ACGI. Certain parameters can be used to measure the similarity between images. Here, SSIM (Structure similarity index) is calculated between consecutive frames to estimate similarity. Fig. 5 represents graphs of calculated SSIM of scintillated datasets. ACGI output sequences lead SSIM of consecutive frames near to 0.95 which is better compared to CGI and turbulent sequences. Overall comparison depicts that the proposed approach outperforms state of the art methods.

\section{Conclusion and Future Work}

This paper introduces a novel approach to restore a high-quality image from a video sequence degraded by atmospheric turbulence. Time and computational cost of the proposed approach are proportional to the heat scintillation. This approach modifies the CGI by changing reference frame selection technique and converting blind iteration using threshold value. Registration is performed between target frames with updated consequent reference frames. Experiments with degraded sequences show the more accurate performance compare to the existing method. Measured parameters clearly represent the superiority of proposed method. In future, the algorithm should also work for real motion detection and restoration. The approach should also work for video sequences containing scene change.

\section{Acknowledgment}

The authors would like to thank Charusat Space Research and Technology Center (CSRTC), and Central Research Laboratory, Bharat Electronics, Bangalore for providing required resources and platform to carry out research work.

\section{REFERENCES}

1. N. Anantrasirichai, A. Achim, N. G. Kingsbury and D. R. Bull, Atmospheric Turbulence Mitigation Using Complex Wavelet-Based Fusion, IEEE Transactions on Image Processing, vol. 22, no. 6, pp. 2398-2408, June 2013.

2. D. H. Frakes, A New Method for Registration-Based Medical Image Interpolation, IEEE Transactions on Medical Imaging, vol. 27, no. 3, pp. 370-377, March 2008.

3. X. Zhu, P. Milanfar, Image Reconstruction from Videos Distorted by Atmospheric Turbulence, Proc. SPIE Electronic Imaging Conf. Visual Information Processing and Comm., Jan. 2010.

4. W. Zhang, Distortion-driven Turbulence Image Blur Effect Removal using Variational Model and Kernel Regression, 2014 International Conference on Identification, Information and Knowledge in the Internet of Things, Beijing, pp. 8-8, 2014.

5. X. Zhu, P. Milanfar, Stabilizing and deblurring atmospheric turbulence, IEEE International Conference on Computational Photography (ICCP), Pittsburgh-USA, pp. 1-8, 2011.

6. Y. Lou, S. H. Kang, S. Soatto, A. L. Bertozzi, Video stabilization of atmospheric turbulence distortion, Inverse Problems in Imaging, Special Issue in honor of Tony Chan,vol. 7, no. 3, pp. 839 - 861, August 2013.

7. K. Parmar, D. Israni, A. Shah, An efficient technique for subpixel accuracy using integrated feature based image registration, 2nd International Conference for Convergence in Technology (I2CT), Pune-India, pp. 271-276, 2017.

8. Y. Chen, J. Wu, G. Yu, Adaptive Proximal Point Algorithms for Total Variation Image Restoration, Statistics, Optimization and Information Computing, Vol. 3, no. 1, pp 15C29, March 2015.

9. R. Chokshi, D. Israni and N. Chavda, An efficient deconvolution technique by identification and estimation of blur, 2016 IEEE International Conference on Recent Trends in Electronics, Information Communication Technology (RTEICT), Bangalore, pp. 1723, 2016.

10. D. Fraser, G. Thorpe, and A. Lambert, Atmospheric turbulence visualization with wide-area motion-blur restoration, Journal of the Optical Society of America A, vol. 16, no. 7, pp. 1751-1758, 1999.

11. J. Kybic, M. Unser, Fast parametric elastic image registration, IEEE Transactions on Image Processing, vol. 12, no. 11, pp. 1427-1442, Nov. 2003.

12. B. Fishbain, P. Leonid, Yaroslavsky, Ianir Ideses Spatial, Temporal, and Interchannel Image Data Fusion for Long-Distance Terrestrial Observation Systems, Advances in Optical Technologies, vol. 2008, Article ID 546808, pp. 1-18, 2008.

13. D. Kamenetsky, M. Zucchi, G. Nichols, D. Booth and A. Lambert, Interactive Atmospheric Turbulence Mitigation, International Conference on Digital Image Computing: Techniques and Applications (DICTA), Gold Coast, QLD, pp. 1-8, 2016.

14. M. Moklyachuk, M. Sidei, Interpolation Problem for Stationary Sequences with Missing Observations, Statistics Optimization and Information Computing, Vol. 3, no. 3, pp 259C275, September 2015. 
15. M. Luz, M. Moklyachuk, Minimax Interpolation Problem for Random Processes with Stationary Increments, Statistics, Optimization and Information Computing, vol. 3, no. 1, March 2015, pp 30C41.

16. G. J. Sullivan, R. L. Baker, Motion compensation for video compression using control grid interpolation, International Conference on Acoustics, Speech, and Signal Processing (ICASSP), Toronto, Canada, pp. 2713-2716, 1991.

17. D. H. Frakes, J. W. Monaco and M. J. T. Smith Suppression of atmospheric turbulence in video using an adaptive control grid interpolation approach, 2001 IEEE International Conference on Acoustics, Speech, and Signal Processing. Proceedings (Cat No.01CH37221), Salt Lake City, UT, 2001, pp. 1881-1884 vol.3.

18. J Gilles, N. B. Ferrante, Open Turbulent Image Set (OTIS), Journal of Pattern Recognition Letters, vol. 86, pp. 38-41, January 2017.

19. J. Ashburner J, K.J. Friston, Spatial transformation of images, Human Brain Function, edited by Frackowiak RSJ, Friston KJ, Frith CD, Dolan RJ, and Mazziotta JC, Academic Press, San Diego, London, pp. 43C58, 1997.

20. R. Abdoola, G. Noel, B. v. Wyk and E. Monacelli, Correction of Atmospheric Turbulence Degraded Sequences Using Grid Smoothing, 8th International Conference on Image Analysis and Recognition (ICIAR), Burnaby, BC, Canada, June 2011.

21. A. Pathak, A. Chaturvedi, Estimation of the reliability function for two-parameter exponentiated Rayleigh or Burr type X distribution, Statistics, Optimization and Information Computing, vol. 2, no. 4, pp. 305-322, December 2014. 